\section{Canine perineal hernia: a review of 44 cases}

\section{Stefanos Kladakis ${ }^{1}$, Konstantinos Terpsidis', Anastasia Komnenou ${ }^{2}$, Andreas Kyriazis $^{3}$, John Vagiatis ${ }^{1}$}

3rd Army Veterinary Hospital, Thermi, Thessaloniki, Greece

Aristotle University, Companion Animal Clinic, Thessaloniki, Greece

Small Animal Veterinary Practice, Thessaloniki, Greece

This study examined complete case records of dogs presented to our practice who were diagnosed and treated for perineal hernia (PH) over a 7-year period (2005-2012) and reports short term and long term clinical outcome of these cases following treatment.

Forty four dogs with a total of $56 \mathrm{PH}$ were reviewed. All dogs underwent surgery and were treated with a combination of anatomic reapposition of the pelvic diaphragm (PD) along with internal obturator muscle transposition (IOMT). In dogs with bilateral $\mathrm{PH}$ the second herniorrhaphy was performed with 4 weeks interval.

Breeds most commonly presented were German Shepherds (25\%) and Mongrels (18.18\%). Common causes of presentation were perineal swelling $(84.1 \%)$, tenesmus $(77.3 \%)$ and dyschezia (36.4\%). Clinical examination findings revealed that $\mathrm{PH}$ was unilateral (72.7\%) or bilateral (27.3\%). Unilateral hernias were categorized as right $(68,75 \%)$ or left $(31.25 \%)$. Prostate enlargement was further diagnosed in 17 (38.6\%) dogs. During surgery hernial contents were retroperitoneal fat $(69,64 \%)$, rectum $(33.9 \%)$, prostate $(14.28 \%)$, urinary bladder (7.14\%) and small intestine (1.78\%). Castration was performed in the same anesthetic episode to 42 dogs. One dog was already castrated a year before $\mathrm{PH}$ was diagnosed and one dog wasn't castrated due to owner's related reasons. Colopexy was performed in 2 dogs (4.54\%) prior to $\mathrm{PH}$ repair. Postoperatively follow-up was performed initially with clinical examination and afterwards owners and referring vets were contacted via telephone. Mean follow-up time was 21.09 months (range 6-36 months). $\mathrm{PH}$ was resolved in 43 dogs $(97.72 \%)$. One dog had recurrence 5 months after initial surgery but the owners were reluctant to further surgical intervention. Wound complications occurred in 6 cases $(10.71 \%)$ within the first 30 days after surgery. Postoperative fecal straining persisted in 13 dogs (29.54\%) and finally resolved in all patients 2-8 months after surgery (mean time 5.6 months). The outcome was recorded as excellent in 35 dogs (79.54\%), good in 6 dogs (13.63\%), fair in 2 dogs (4.54\%) and poor in 1 $\operatorname{dog}(2.27 \%)$.

Although in the literature numerous techniques have been utilized to treat $\mathrm{PH}$, a combination of $\mathrm{PD}$ anatomic reapposition along with IOMT should be considered as the preferred method of surgical repair.

\section{Evaluation of an axial pattern flap based on the cranial cutaneous branch of the saphenous artery}

\section{Andrew Murdoch'1, Sam Greenaway², Alan Danielski ${ }^{3}$}

The Queen's Veterinary School Hospital, University of Cambridge, Madingley Road, Cambridge CB3 OES, Cambridge, UK

Scott Veterinary Clinic, Goldington Road, Bedford MK41 ODS, Bedford, UK

Davies Veterinary Specialists, Manor Farm Business Park, Higham Gobion, Hertfordshire SG5 3HR, Hertfordshire, UK

\section{BACKGROUND}

Axial pattern skin flaps are subdermal plexus flaps designed to include a direct cutaneous artery for wound reconstruction. An axial pattern flap based on the cranial cutaneous branch of the saphenous artery (CCSA) of the dog was previously described in a case report. The angiosome of the CCSA has not been investigated to determine whether this axial pattern flap is clinically applicable beyond a single case report.

\section{OBJECTIVE}

To determine the borders of an axial pattern flap based on the CCSA in dogs.

\section{STUDY DESIGN}

Cadaveric anatomic study.

\section{METHOD}

Ten Greyhound dogs were administered intravenous heparin immediately prior to euthanasia for reasons unrelated to this study, complying with ethical and welfare guidelines. The medial thighs were clipped and palpable anatomic landmarks outlined with a skin marker. A caudomedial skin incision and blunt dissection allowed identification and isolation of the CCSA. All other branches of the saphenous artery were ligated prior to infusion of methylene blue (left pelvic limbs) or iohexol (right pelvic limbs) into the CCSA via a cannula. The medial skin was freed from the thigh for photographic and radiographic studies to be performed, to assess skin perfusion.

\section{RESULTS}

The CCSA was reliably identified in all dogs branching from the saphenous artery as it became superficial to the biceps femoris muscle, immediately distal to the caudal cutaneous branch and proximal to the genicular branches, which were variably paired or singular. Methylene blue studies demonstrated perfusion of the skin on the medial thigh, extending proximally to anastomoses with superficial cutaneous branches of the deep circumflex iliac artery, cranially to the cranial border of the thigh and distally to the level of the tibial plateau. This pattern was well correlated with the radiographic assessment of vessel arborisation. 


\section{Congress 2015}

\section{CONCLUSIONS}

The CCSA angiosome suitable for use as an axial pattern skin flap was defined as the area cranial to the saphenous artery and caudal to the cranial border of the thigh, extending proximally from the level of the tibial tuberosity to two-thirds of the distance to the inguinal ring. This conservatively estimated the overall angiosome of the CCSA to allow easy tension-free closure of the donor site for clinical usage.

\section{CLINICAL SIGNIFICANCE}

An axial pattern flap based on the CCSA offers an alternative means of reconstruction of wounds in the region of the stifle of the dog.

\section{Clinical parameters in bitches with open versus closed pyometra}

\section{Supranee Jitpean ${ }^{1,2}$, Ulf Emanuelson', Ann Pettersson', Bodil Ström Holst' ${ }^{1}$, Odd Höglund', Ragnvi Hagman'}

Swedish University of Agricultural Sciences, Uppsala, Sweden Khon Kaen University, Khon Kaen, Thailand

\section{INTRODUCTION AND AIM}

Pyometra is a common and potentially life-threatening disease in dogs. Clinical signs include depression, polyuria, polydipsia, anorexia and vaginal discharge (open cervix pyometra) but vaginal discharge can sometimes be absent (closed cervix pyometra). Closed cervix pyometra is considered to be more severe. Leukocytosis, neutrophilia with left shift, anaemia and hypoalbuminaemia are common findings in dogs with pyometra and leukocytosis and anaemia are more common in closed pyometra. The aim of this study was to compare clinical and hematological variables between bitches with closed and open pyometra.

\section{MATERIALS AND METHODS}

Data from 309 bitches diagnosed with pyometra, 237 with open pyometra and 72 with closed pyometra, was studied retrospectively. Case history data, physical examination findings and hematology results were analysed. Prolonged postoperative hospitalization was defined as $\geq 3$ days.
Statistical analyses were performed by using SAS 9.2 for Windows version 6.1.7601 (SAS Institute Inc. Cary, NC, USA). Chi-square tests were used for comparisons between open and closed pyometra.

\section{RESULTS}

In bitches with open pyometra, the mean \pm SD age was $8.2 \pm$ 3.1 years $(n=236)$ and mean \pm SD weight was $24.5 \pm 14.4 \mathrm{~kg}$ ( $n=195)$. In bitches with closed pyometra the mean age was $8.0 \pm 2.7$ years $(n=71)$ and the mean weight was $21.8 \pm 14.1$ $\mathrm{kg}(\mathrm{n}=67)$.

Leukocytosis was present in $57 \%$ and anaemia in $53 \%$ of the bitches with open pyometra, and only $4 \%$ had leukopenia in this group. The corresponding proportions for bitches with closed pyometra were $46 \%$ for leukocytosis, $40 \%$ for anaemia and $4 \%$ for leukopenia.

In bitches with open pyometra, moderate to severe depression was recorded in $26 \%$, vomiting in $19 \%$, polydipsia in $62 \%$ and polyuria in $61 \%$. In cases with closed pyometra, moderate to severe depression was recorded in $28 \%$, vomiting in $25 \%$, polydipsia in $59 \%$ and polyuria in $52 \%$.

The differences in distribution of analysed variables were not significantly different between the two groups ( $p>0.05)$

Of the bitches with open pyometra, the postoperative hospitalization was prolonged in $21 \%$ whereas the corresponding proportion in bitches with closed pyometra was $18 \%(p=0.50)$.

\section{CONCLUSIONS}

None of the studied variables differed significantly in bitches with open versus bitches with closed pyometra

\section{lleocolic valve resection in dogs and cats: 17 cases (2001-2014)}

\section{Yordan Fernandez, Mayank Seth, Daniela Murgia, Jordi Puig}

Animal Health Trust, Newmarket, UK

\section{INTRODUCTION}

There are only occasional reports of dogs or cats with resection of the ileocolic valve (ICV) described in the veterinary literature. The aims of this retrospective study were to characterise the different diseases affecting the ICV resulting in surgical resection and to determine the long-term outcome in a cohort of dogs and cats.

\section{MATERIAL AND METHODS}

Medical records of dogs and cats that had the ICV removed between 2001 and 2014 at a multidisciplinary referral hospital were retrospectively reviewed. Data regarding signalment, diagnosis and long-term management were obtained. 